\title{
Geostatistical Approach for Spatial Interpolation of Meteorological Data
}

\author{
DERYA OZTURK ${ }^{1}$ and FATMAGUL KILIC ${ }^{2}$ \\ ${ }^{1}$ Department of Geomatics Engineering, Ondokuz Mayis University, Kurupelit Campus, 55139, Samsun, Turkey \\ ${ }^{2}$ Department of Geomatics Engineering, Yildiz Technical University, Davutpasa Campus, 34220, Istanbul, Turkey
}

Manuscript received on February 2, 2015; accepted for publication on March 1, 2016

\begin{abstract}
Meteorological data are used in many studies, especially in planning, disaster management, water resources management, hydrology, agriculture and environment. Analyzing changes in meteorological variables is very important to understand a climate system and minimize the adverse effects of the climate changes. One of the main issues in meteorological analysis is the interpolation of spatial data. In recent years, with the developments in Geographical Information System (GIS) technology, the statistical methods have been integrated with GIS and geostatistical methods have constituted a strong alternative to deterministic methods in the interpolation and analysis of the spatial data. In this study; spatial distribution of precipitation and temperature of the Aegean Region in Turkey for years 1975, 1980, 1985, 1990, 1995, 2000, 2005 and 2010 were obtained by the Ordinary Kriging method which is one of the geostatistical interpolation methods, the changes realized in 5-year periods were determined and the results were statistically examined using cell and multivariate statistics. The results of this study show that it is necessary to pay attention to climate change in the precipitation regime of the Aegean Region. This study also demonstrates the usefulness of the geostatistical approach in meteorological studies.
\end{abstract}

Key words: geostatistical interpolation, geographic information system, ordinary kriging, meteorological data.

\section{INTRODUCTION}

Measurement and evaluation of the spatially distributed meteorological data have become important in connection with climate-change impact studies, determination of water budgets at different temporal and spatial scales, as well as validation of atmospheric and hydrological models. Meteorological data are usually available from a limited number of meteorological stations (Hofierka et al. 2002), mostly because it is not economically and technically possible to obtain meteorological data throughout the entire surface. For this reason, spatial interpolation of the meteorological variables obtained from the certain sample points is performed in order to create a model for the entire surface.

Spatial interpolation is the procedure of estimating the value of unsampled points using existing observations (Waters 1997). Methods for spatial interpolation can be classified into two main categories

Correspondence to: Derya Ozturk

E-mail: dozturk@omu.edu.tr 
as deterministic and geostatistical (Burrough and McDonnell 1998, Matthews 2002). Deterministic interpolation techniques calculate the values of unsampled points and create surfaces from measured points, based on either the extent of similarity or the degree of smoothing (Matthews 2002). Deterministic methods do not use probability theory (Waters 1997). Geostatistical interpolation techniques use the statistical properties of the measured points, quantify the spatial autocorrelation among the measured points and account for the spatial configuration of the sample points around the estimation location (Matthews 2002).

Kriging is a geostatistical technique for optimal spatial estimation (Waller and Gotway 2004). Kriging provides a solution to the problem of estimation based on a continuous model of stochastic spatial variation and takes the variogram model (Webster and Oliver 2007). Today, with the developments in computer and Geographical Information System (GIS) technologies, the statistical methods have been integrated with GIS and the geostatistical methods have constituted a strong alternative to deterministic methods in the interpolation of the spatial data. In addition, statistical methods to analyze the interpolated layers have allowed a better understanding of the changes occurred in the specific time period.

Climate change is one of the biggest threats for the entire globe (Kropp 2015). Climate changes affect the natural balance of the earth and ecosystems and whole life is disrupted (National Academy of Sciences 2009) Climate change is most often measured by changes in primary climate variables, such as temperature and precipitation. These variables are the main drivers of climate changes (Sheffield and Wood 2012). For this reason, to understand and monitor the changes and their causes and effects accurately, changes should be determined both spatially and quantitatively and the results should be evaluated in detail.

In this study it is aimed to investigate the spatial distribution of precipitation and temperature of the Aegean Region in Turkey for years 1975, 1980, 1985, 1990, 1995, 2000, 2005 and 2010 by the Ordinary Kriging method and statistically examine the results using cell statistics and multivariate statistics to understand the changes. This study demonstrates the usefulness of the geostatistical approach for both interpolation of meteorological data and analysis and comparison of the results.

\section{MATERIALS AND METHODS}

The Aegean Region is one of Turkey's seven geographical regions. It is surrounded by the Aegean Sea on the west and takes its name from the Aegean Sea (Ozcaglar 2014). In this study, the area comprising eight provinces located in the Aegean Region has been analyzed. The total area is approximately 90,000 $\mathrm{km}^{2}$ (Figure 1). The coastal areas of the Aegean Region has a Mediterranean climate. The effects of the Mediterranean climate extend up to 100-150 km inland from the coast. In coastal areas, winters are mild and summers are very hot and dry. The interior side of the region is affected by the continental climate (Sensoy et al. 2008).

In the present study, the time series of monthly precipitation and temperature data from 98 meteorological stations for the years 1975, 1980, 1985, 1990, 1995, 2000, 2005 and 2010 were used. Spatial distributions of the stations are shown in Figure 1. The geospatial interpolation of temperature and precipitation data and all statistical analyses of the precipitation and temperature layers were performed using ArcGIS 10.0 software (Esri, Redlands, CA). The method of creating an estimation surface layer with the Ordinary Kriging is explained in Section "Creating An Estimation Surface Layer with the Ordinary Kriging" and statistical analyses of layers is presented in Section "Statistical Analyses of Layers". 


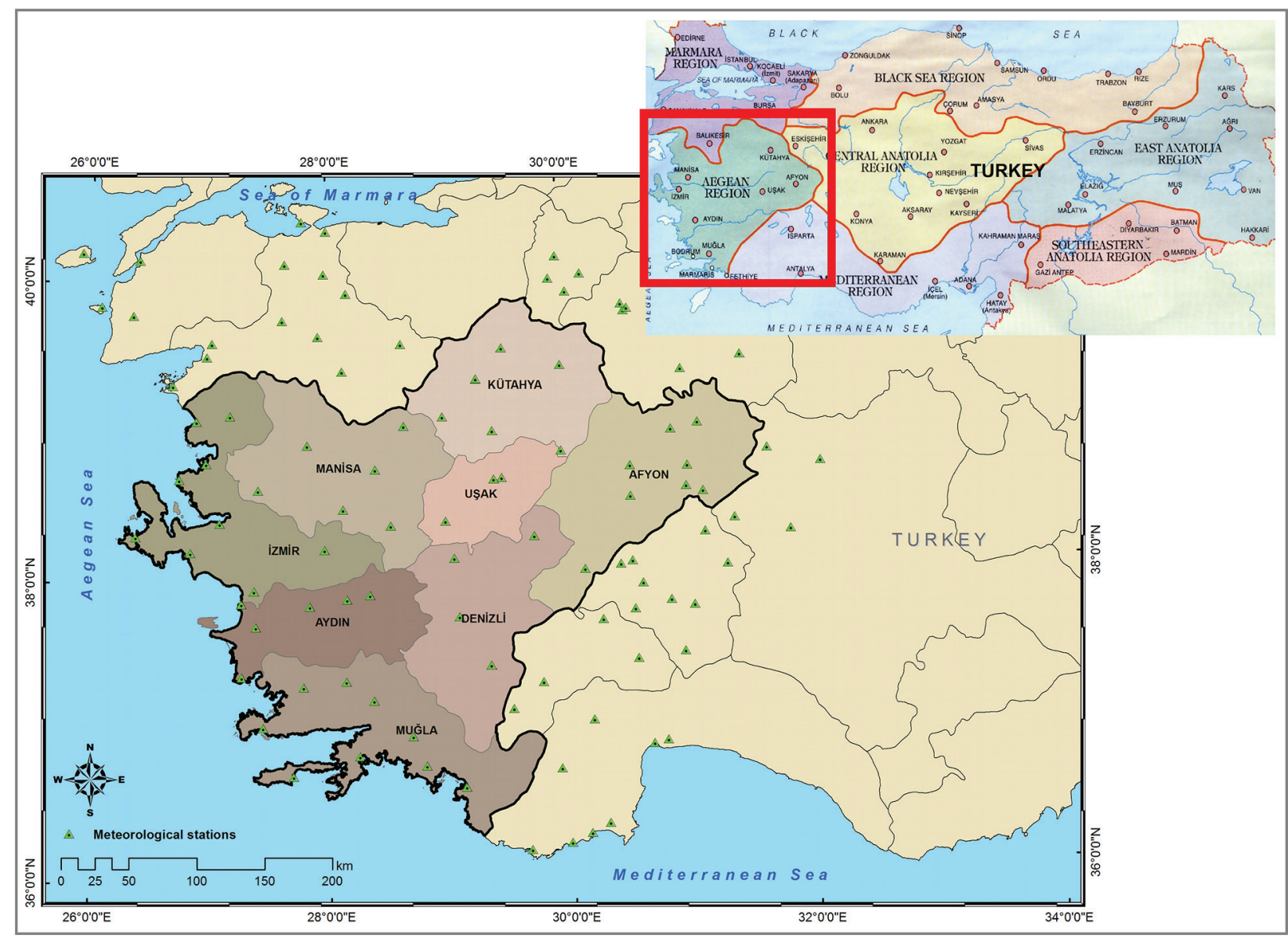

Figure 1 - The location of the study area (The Aegean Region, Turkey) and spatial distributions of the meteorological stations.

\section{CREATING AN ESTIMATION SURFACE LAYER WITH THE ORDINARY KRIGING}

Estimation with the Kriging interpolation method has a two-step process: (i) fitting a model: creation of the variograms and covariance functions to estimate the statistical dependence (spatial autocorrelation) values that depend on the model of autocorrelation and (ii) making an estimation: estimation of the unknown values (ESRI 2014a).

The first step in the Ordinary Kriging is to create a semivariogram from the scatter point set to be interpolated. A semivariogram consists of (i) an empirical semivariogram (experimental variogram) and (ii) a model semivariogram (GMS User Manuel 2012). Semivariogram is a mathematical model of the semivariance as a function of lag and displays the statistical correlation of nearby points (Prasad et al. 2007). Spatial autocorrelation (means feature similarity) is based on both feature locations and feature values simultaneously (not only based on feature locations or attribute values alone). Given a set of features and an associated attribute, it evaluates whether the pattern expressed is clustered, dispersed, or random (Matthews 2002). Empirical semivariogram, computed by (Eq.1) for all pairs of locations separated by distance h (ESRI 2014a):

Semivariogram $($ distance $\mathrm{h})=0.5 *$ average[(value at location $\mathrm{i}-$ value at location $\left.\mathrm{j})^{2}\right]$ 
The formula involves calculating the difference squared between the values of the paired locations. Figure 2 shows the pairing of one point (the red point) with all other measured locations. This process continues for each measured point (ESRI 2014a).

Often, each pair of locations has a unique distance, and there are often many pairs of points. To plot all pairs quickly becomes unmanageable. Instead of plotting each pair, the pairs are grouped into lag bins. The empirical semivariogram is a graph of the averaged semivariogram values on the y-axis and the distance (or lag) on the x-axis (Figure 3) (ESRI 2014a).

When two locations are close to each other (far left on the x-axis of the semivariogram cloud), then they are expected to be similar (low on the y-axis of the semivariogram cloud) (ESRI 2014a, Prasad et al. 2007). "As pairs of locations become farther apart (moving to the right on the x-axis of the semivariogram cloud), they should become more dissimilar and have a higher squared difference (moving up on the y-axis of the semivariogram cloud)" (ESRI 2014a).

Once the empirical variogram is obtained, the next step is to define a model semivariogram (GMS User Manuel 2012). Semivariogram modeling is a main step between spatial description and spatial estimation. The empirical semivariogram provides information on the spatial autocorrelation of datasets, however does not supply information for all possible directions and distances. For this reason, it is necessary to fit

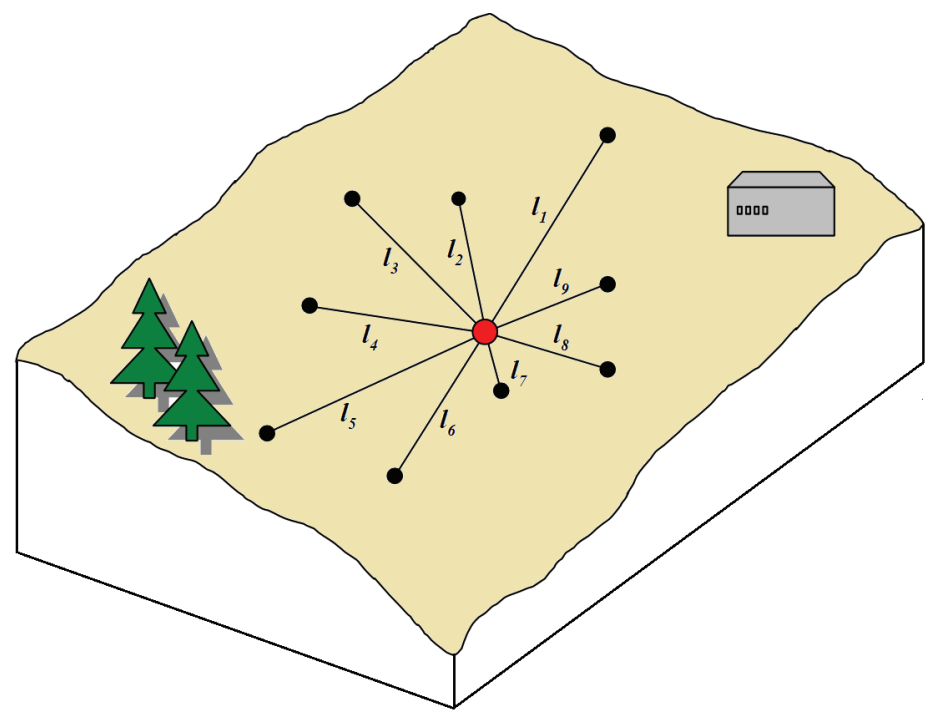

Figure 2 - Calculation of the difference squared between the paired locations.

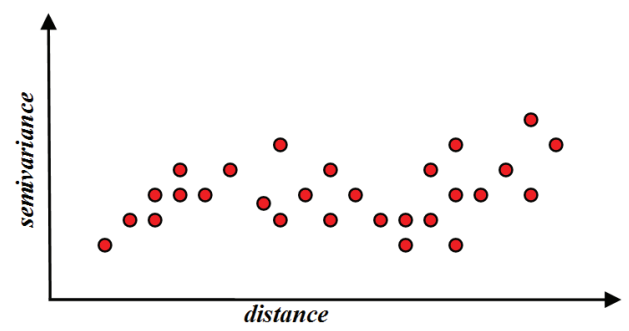

Figure 3 - Empirical semivariogram graph example. 
a model (a continuous function or curve) to the empirical semivariogram (ESRI 2014a). There are many semivariogram models. Some of the most common are linear, circular, spherical, exponential, and Gaussian model (Li and Heap 2008). The selected model influences the estimation of the unknown values and each model is designed to fit different types of phenomena more accurately (ESRI 2014a).

Once the model variogram is obtained, it is used to calculate the weights used in Kriging (GMS User Manuel 2012). The basic equation used in the Ordinary Kriging is as (Eq.2) (ESRI 2014a, GMS User Manuel 2012, Borga and Vizzaccaro 1996):

$$
\hat{Z}\left(s_{0}\right)=\sum_{i=1}^{N} \lambda_{i} Z\left(s_{i}\right)
$$

Where;

$Z\left(s_{i}\right):$ the measured value at the $i$ th location

$\lambda_{i}$ : an unknown weight for the measured value at the $i$ th location

$\left(s_{0}\right)$ : the estimation location

$\mathrm{N}$ : the number of measured values

With Kriging method, the value $\hat{Z}\left(s_{0}\right)$ at the point $s_{0}$, where the true unknown value is $Z\left(s_{0}\right)$, is estimated by a linear combination of the values at $\mathrm{N}$ surrounding data points (Borga and Vizzaccaro 1996).

In the Ordinary Kriging, the weight, $\lambda_{i}$, depends on a fitted model to the measured points, the distance to the estimation point, and the spatial relationships among the measured values around the estimation location (ESRI 2014a) and the Kriging weights are calculated by minimizing the variance (Li and Heap 2008). The Ordinary Kriging is the most widely used Kriging method (Wackernagel 2003) and this method assumes that the data set has a stationary variance but also a non-stationary mean value within the search radius. The Ordinary Kriging is highly reliable and recommended for most data sets (Vertical Mapper User Guide 2008).

\section{STATISTICAL ANALYSES OF LAYERS}

\section{CELL STATISTICS}

In a local function, the value at each location on the output raster is a function of the input values at that location. When computing a local function, input rasters can be combined and a statistic can be calculated. In ArcGIS software, several cell statistics can be calculated for raster layers: (i) MEAN: Calculates the mean (average) of the inputs, (ii) MAXIMUM: Determines the maximum (largest value) of the inputs, (iii) MEDIAN: Calculates the median of the inputs, (iv) MINIMUM: Determines the minimum (smallest value) of the inputs, (v) RANGE: Calculates the range (difference between largest and smallest value) of the inputs, (vi) STD: Calculates the standard deviation of the inputs (ESRI 2014b).

\section{Multivariate Statistics}

The multivariate statistics allow exploration of relationships between many different data layers or types of attributes. In band collection function, main statistical measures (minimum, maximum, mean and standard deviation) can be calculated for every layer and in addition to these standard statistics, the covariance and correlation matrices can also be determined (ESRI 2014b). 


\section{RESULTS AND DISCUSSION}

The time series of monthly precipitation and temperature data for the years 1975, 1980, 1985, 1990, 1995, 2000, 2005 and 2010 were used for preparing spatial distribution layers of precipitation and temperature of the Aegean Region, Turkey. The Ordinary Kriging interpolation was applied for each month and a total of 192 interpolations were performed (96 for precipitation and 96 for temperature) and grid layers with 250-meter pixel size were formed. The Ordinary Kriging interpolation results of the precipitation and temperature data for January are shown in Figures 4 and 5, respectively.

Based on the multivariate statistics (band collection), spatial analyses were applied for the monthly precipitation and temperature layer series which calculated by the Ordinary Kriging. Table I (for precipitation) and Table II (for temperature) represent the main statistics, including the minimum, maximum, mean and standard deviation values. In addition, the correlation coefficients were calculated with these analyses (Tables III and IV).

When examining Table I, it was seen that the highest "average precipitation" and the highest "precipitation" values were in December 1990. Table II shows that both highest "average temperature" and highest "temperature" values were in August 2010.

According to Table III, correlation coefficients for precipitation are between -0.04847 and 0.92382 for January, 0.18609 and 0.92908 for February, -0.57255 and 0.74793 for March, -0.43776 and 0.76531 for April, -0.62850 and 0.86128 for May, -0.26385 and 0.79177 for June, -0.22994 and 0.74375 for July, 0.01302 and 0.80480 for August, -0.20455 and 0.69922 for September, -0.68396 and 0.74704 for October,
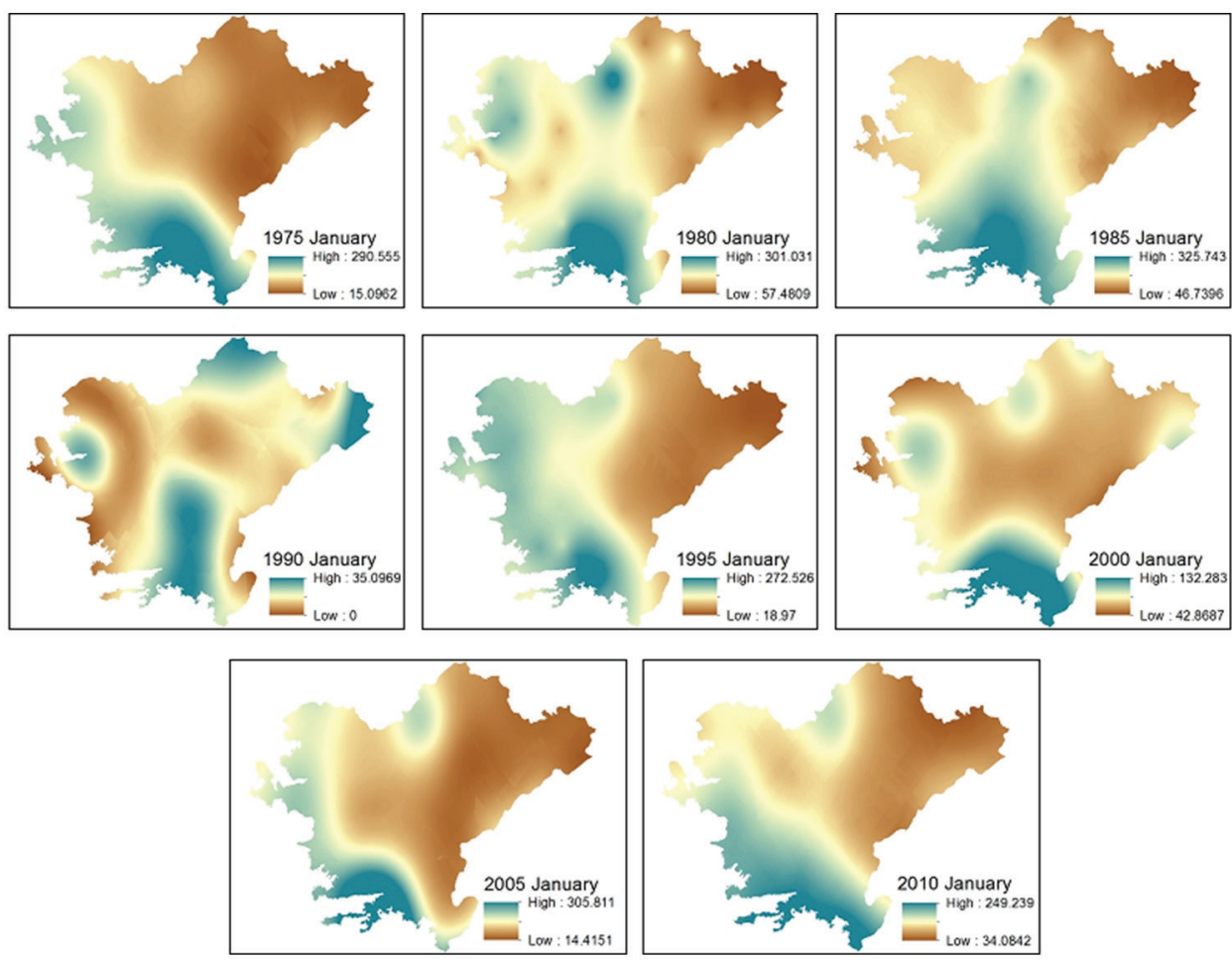

Figure 4 - The Ordinary Kriging interpolation results of the precipitation data for the month of January (For the years of 1975, 1980, 1985, 1990, 1995, 2000, 2005, and 2010). 

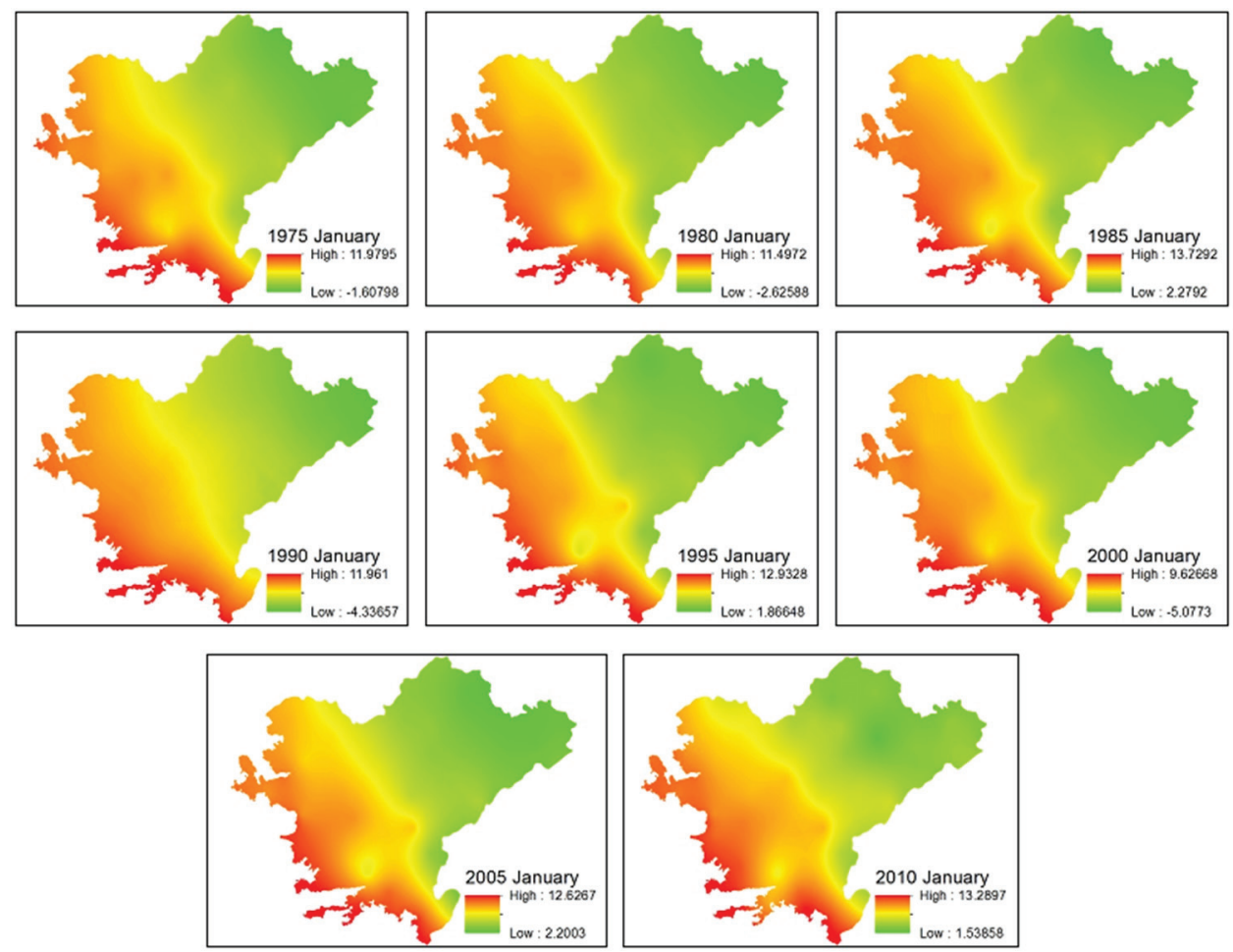

Figure 5 - The Ordinary Kriging interpolation results of the temperature data for the month of January (For the years of 1975, 1980, 1985, 1990, 1995, 2000, 2005, and 2010).

0.29150 and 0.85486 for November, 0.22520 and 0.90808 for December. According to Table IV, correlation coefficients for temperature are between 0.95152 and 0.99524 for January, 0.95804 and 0.99591 for February, 0.91016 and 0.99459 for March, 0.94823 and 0.99361 for April, 0.96744 and 0.99414 for May, 0.94961 and 0.99084 for June, 0.93391 and 0.99041 for July, 0.94175 and 0.99293 for August, 0.96714 and 0.99254 for September, 0.97214 and 0.99542 for October, 0.93409 and 0.99328 for November, 0.96537 and 0.99665 for December.

Correlations above 0.80 generally are accepted as high correlations. Correlations between 0.50 and 0.80 are usually considered as medium (moderate) correlations and correlations below 0.50 are typically regarded as low correlations (Wang et al. 1990). Accordingly, very high correlation values were observed between temperature values of years 1975, 1980, 1985, 1990, 1995, 2000, 2005 and 2010 for all months (Table IV). But, the correlations between layers of precipitation were examined, both high and low correlation values were observed. For precipitation layers, the highest correlation was observed between the year of 1975 and 2010 for January; 2005 and 2010 for February; 1985 and 1995 for March; 2005 and 2010 for April; 1990 and 2000 for May; 1990 and 1995 for June; 1975 and 2010 for July; 1975 and 2000 for August; 1980 and 2000 for September; 2000 and 2010 for October; 2005 and 2010 for November; 1980 and 1990 for December.

By calculating cell statistics, a statistic for each cell in an output raster can be calculated based on the values of multiple input rasters (ESRI 2014b). In this study; maximum, minimum, mean, median, range and standard deviation layers were produced by using precipitation and temperature layers for years 1975, 1980, 1985, 1990, 1995, 2000, 2005 and 2010 for all months. Totally 144 statistical layers were obtained 
TABLE I

Main statistics for precipitation layers (Minimum, Maximum, Mean, Standard deviation).

\begin{tabular}{|c|c|c|c|c|c|c|c|c|c|c|c|}
\hline \multicolumn{12}{|c|}{ STATISTICS of INDIVIDUAL LAYERS - PRECIPITATION } \\
\hline \multicolumn{2}{|c|}{ Layer(Year) } & \multirow{2}{*}{$\frac{\text { MIN }}{15.0962}$} & \multirow{2}{*}{$\frac{\text { MAX }}{290.5551}$} & \multirow{2}{*}{$\begin{array}{c}\text { MEAN } \\
105.2915\end{array}$} & \multirow{2}{*}{$\begin{array}{c}\text { STD } \\
68.9066\end{array}$} & \multicolumn{2}{|c|}{ Layer(Year) } & \multirow{2}{*}{$\begin{array}{c}\text { MIN } \\
0.0000\end{array}$} & \multirow{2}{*}{$\frac{\text { MAX }}{11.4688}$} & \multirow{2}{*}{$\frac{\text { MEAN }}{3.8647}$} & \multirow{2}{*}{$\begin{array}{r}\text { STD } \\
3.2307\end{array}$} \\
\hline January & 1975 & & & & & July & 1975 & & & & \\
\hline & 1980 & 57.4809 & 301.0310 & 144.8527 & 41.7206 & & 1980 & 0.0000 & 6.4559 & 1.5206 & 0.9994 \\
\hline & 1985 & 46.7396 & 325.7432 & 160.8042 & 58.6881 & & 1985 & 0.0000 & 7.6697 & 0.7246 & 0.7361 \\
\hline & 1990 & 0.0000 & 35.0969 & 12.1264 & 5.1998 & & 1990 & 0.0000 & 39.4809 & 5.0383 & 5.5698 \\
\hline & 1995 & 18.9700 & 272.5263 & 120.9055 & 59.4265 & & 1995 & 0.0000 & 189.5152 & 34.9476 & 30.6981 \\
\hline & 2000 & 42.8687 & 132.2835 & 69.6099 & 16.4874 & & 2000 & 0.0000 & 23.2685 & 3.9946 & 4.4414 \\
\hline & 2005 & 14.4151 & 305.8113 & 70.4621 & 46.7614 & & 2005 & 0.0000 & 85.0155 & 24.6473 & 19.6237 \\
\hline & 2010 & 34.0842 & 249.2391 & 114.5032 & 48.2253 & & 2010 & 0.0173 & 12.8247 & 5.7179 & 3.1505 \\
\hline \multirow[t]{8}{*}{ February } & 1975 & 35.9389 & 164.3231 & 58.1565 & 23.4616 & August & 1975 & 0.0000 & 66.0855 & 12.1734 & 13.2959 \\
\hline & 1980 & 11.4949 & 100.1111 & 35.0220 & 14.2885 & & 1980 & 0.0000 & 29.5959 & 4.3619 & 5.3946 \\
\hline & 1985 & 29.6399 & 149.1992 & 73.9867 & 26.1003 & & 1985 & 0.0000 & 41.5747 & 10.0517 & 7.4810 \\
\hline & 1990 & 7.0911 & 143.2509 & 49.7590 & 25.1979 & & 1990 & 0.4801 & 67.0008 & 10.8518 & 7.5046 \\
\hline & 1995 & 6.6114 & 117.6134 & 27.3189 & 15.0847 & & 1995 & 0.2385 & 16.9660 & 7.1374 & 3.8129 \\
\hline & 2000 & 49.3334 & 109.7159 & 86.5541 & 15.9437 & & 2000 & 0.0000 & 26.2174 & 6.2557 & 5.7277 \\
\hline & 2005 & 18.2838 & 330.9904 & 117.0124 & 68.6871 & & 2005 & 0.4208 & 17.9898 & 8.6525 & 4.3138 \\
\hline & 2010 & 47.3964 & 343.1137 & 151.4875 & 62.9992 & & 2010 & 0.0000 & 20.6179 & 7.7861 & 5.9152 \\
\hline \multirow{8}{*}{ March } & 1975 & 26.2257 & 107.8506 & 64.0406 & 15.5155 & September & 1975 & 0.0000 & 49.7015 & 10.9055 & 10.0935 \\
\hline & 1980 & 49.5541 & 159.2850 & 95.6635 & 22.8128 & & 1980 & 0.0000 & 55.4421 & 16.4988 & 14.7347 \\
\hline & 1985 & 27.8837 & 106.6610 & 59.4646 & 17.9716 & & 1985 & 0.0000 & 6.5261 & 1.9362 & 1.3202 \\
\hline & 1990 & 6.9016 & 39.8629 & 23.6062 & 5.8286 & & 1990 & 1.4236 & 38.1317 & 20.5715 & 8.0680 \\
\hline & 1995 & 61.6728 & 222.2994 & 126.3867 & 33.2200 & & 1995 & 0.0000 & 46.1658 & 17.5078 & 11.7651 \\
\hline & 2000 & 38.4527 & 143.0037 & 88.8729 & 20.7716 & & 2000 & 0.0000 & 22.9868 & 5.9550 & 5.8907 \\
\hline & 2005 & 33.6981 & 117.8575 & 79.2205 & 15.7518 & & 2005 & 4.7207 & 35.6864 & 15.2393 & 6.0027 \\
\hline & 2010 & 2.0541 & 66.2231 & 30.9691 & 13.4006 & & 2010 & 22.0556 & & & 4.0500 \\
\hline \multirow[t]{8}{*}{ April } & 1975 & 18.2819 & 98.7922 & 51.6425 & 12.2515 & October & 1975 & 9.5328 & 90.6642 & 33.0401 & 13.0100 \\
\hline & 1980 & 29.4372 & 69.5153 & 47.0104 & 7.3046 & & 1980 & 4.3897 & 46.2036 & 22.3987 & 9.1381 \\
\hline & 1985 & 2.0838 & 44.1912 & 19.7078 & 9.1412 & & 1985 & 11.1300 & 151.5788 & 42.3043 & 26.9946 \\
\hline & 1990 & 19.9863 & 90.5741 & 54.8812 & 12.3692 & & 1990 & 7.2120 & 42.6564 & 24.6231 & 8.0837 \\
\hline & 1995 & 18.1657 & 89.0869 & 51.9969 & 14.4514 & & 1995 & 6.0607 & 56.4756 & 34.5488 & 14.9560 \\
\hline & 2000 & 26.9695 & 152.4105 & 85.1713 & 26.0013 & & 2000 & 3.6411 & 98.6216 & 34.3385 & 19.8806 \\
\hline & 2005 & 15.9945 & 90.1445 & 39.7732 & 13.9404 & & 2005 & 11.2323 & 121.7506 & 33.1998 & 17.9017 \\
\hline & 2010 & 9.5546 & 83.4186 & 37.2400 & 14.8342 & & 2010 & 49.5620 & 311.1042 & 116.5370 & 49.2287 \\
\hline \multirow[t]{8}{*}{ May } & 1975 & 39.9216 & 119.2508 & 71.7452 & 17.9338 & November & 1975 & 39.6952 & 239.7979 & 112.4898 & 38.7931 \\
\hline & 1980 & 10.6646 & 77.5801 & 36.4267 & 10.0239 & & 1980 & 31.0222 & 174.3634 & 93.1593 & 26.5030 \\
\hline & 1985 & 1.4462 & 92.5336 & 33.3793 & 9.2920 & & 1985 & 28.2310 & 180.9006 & 85.0800 & 32.2572 \\
\hline & 1990 & 0.0000 & 62.5613 & 23.3644 & 13.0269 & & 1990 & 13.8738 & 74.1966 & 32.1743 & 11.6332 \\
\hline & 1995 & 0.0000 & 76.9429 & 27.1503 & 9.6845 & & 1995 & 31.6046 & 177.6392 & 88.1167 & 27.7271 \\
\hline & 2000 & 1.0087 & 79.3443 & 31.0651 & 20.8717 & & 2000 & 1.8513 & 201.2959 & 54.5331 & 44.2754 \\
\hline & 2005 & 6.2719 & 83.2165 & 44.0559 & 17.0640 & & 2005 & 43.2833 & 254.8676 & 114.4541 & 41.6780 \\
\hline & 2010 & 12.1662 & 46.3693 & 26.6590 & 6.6865 & & 2010 & 8.2926 & 51.3080 & 28.8157 & 9.8915 \\
\hline \multirow[t]{8}{*}{ June } & 1975 & 15.4860 & 100.2484 & 50.0390 & 17.3824 & December & 1975 & 39.7735 & 188.7916 & 88.9672 & 25.1946 \\
\hline & 1980 & 0.0000 & 80.8975 & 24.9794 & 12.4476 & & 1980 & 28.2021 & 296.8829 & 147.5694 & 61.1975 \\
\hline & 1985 & 0.0000 & 45.2143 & 16.5010 & 11.0090 & & 1985 & 19.9052 & 135.5423 & 51.3575 & 16.5321 \\
\hline & 1990 & 0.0000 & 54.2057 & 20.5578 & 14.1597 & & 1990 & 43.9064 & 361.6299 & 165.4436 & 71.0610 \\
\hline & 1995 & 0.0021 & 47.5019 & 9.5297 & 10.6071 & & 1995 & 24.6129 & 165.1980 & 71.9208 & 30.6091 \\
\hline & 2000 & 0.0000 & 37.4081 & 11.7682 & 8.3772 & & 2000 & 14.4990 & 329.9915 & 52.2345 & 36.1115 \\
\hline & 2005 & 0.3222 & 58.3159 & 27.5195 & 12.8268 & & 2005 & 11.9330 & 203.2546 & 65.3737 & 31.7432 \\
\hline & 2010 & 0.7233 & 141.3197 & 55.6312 & 24.9145 & & 2010 & 59.0785 & 231.4372 & 119.4989 & 34.7257 \\
\hline
\end{tabular}


TABLE II

Main statistics for temperature layers (Minimum, Maximum, Mean, Standard deviation).

\begin{tabular}{|c|c|c|c|c|c|c|c|c|c|c|c|}
\hline \multicolumn{12}{|c|}{ STATISTICS of INDIVIDUAL LAYERS - TEMPERATURE } \\
\hline \multicolumn{2}{|c|}{ Layer(Year) } & MIN & MAX & MEAN & STD & Layer(Year) & & MIN & MAX & MEAN & STD \\
\hline \multirow[t]{8}{*}{ January } & 1975 & -1.6080 & 11.9795 & 3.7326 & 3.1861 & July & 1975 & 20.8040 & 28.7429 & 25.1050 & 2.1311 \\
\hline & 1980 & -2.6259 & 11.4972 & 3.0345 & 3.4320 & & 1980 & 21.6467 & 29.0423 & 25.5491 & 1.7273 \\
\hline & 1985 & 2.2792 & 13.7292 & 7.0922 & 2.9161 & & 1985 & 18.8785 & 28.5773 & 24.1568 & 2.6798 \\
\hline & 1990 & -4.3366 & 11.9610 & 2.5856 & 3.4689 & & 1990 & 20.9858 & 29.3868 & 25.8605 & 2.2016 \\
\hline & 1995 & 1.8665 & 12.9328 & 6.2080 & 2.7793 & & 1995 & 19.5084 & 29.1838 & 24.6306 & 2.9346 \\
\hline & 2000 & -5.0773 & 9.6267 & 1.1922 & 3.7585 & & 2000 & 22.6168 & 30.2668 & 27.1807 & 2.0244 \\
\hline & 2005 & 2.2003 & 12.6267 & 6.3250 & 2.6454 & & 2005 & 21.7852 & 29.3529 & 26.1972 & 2.1397 \\
\hline & 2010 & 1.5386 & 13.2897 & 6.6969 & 2.7832 & & 2010 & 21.6157 & 30.2688 & 26.5241 & 2.1923 \\
\hline \multirow[t]{8}{*}{ February } & 1975 & -0.4336 & 11.9043 & 4.1513 & 3.0501 & August & 1975 & 20.1038 & 27.2521 & 23.9362 & 1.9830 \\
\hline & 1980 & -0.3540 & 11.8185 & 4.6182 & 2.9815 & & 1980 & 20.3963 & 28.3603 & 24.5688 & 2.0282 \\
\hline & 1985 & -4.1206 & 10.0607 & 1.7981 & 3.7145 & & 1985 & 21.4755 & 28.3776 & 25.3309 & 1.8210 \\
\hline & 1990 & 0.0275 & 12.6155 & 5.6012 & 3.1362 & & 1990 & 19.6488 & 27.6955 & 24.3883 & 2.2268 \\
\hline & 1995 & 3.9147 & 13.8931 & 7.8196 & 2.5229 & & 1995 & 20.3723 & 28.2541 & 24.5760 & 2.0307 \\
\hline & 2000 & -1.8539 & 11.9216 & 4.6025 & 3.3023 & & 2000 & 20.0756 & 28.9870 & 25.3378 & 2.4911 \\
\hline & 2005 & 1.0839 & 11.0291 & 5.1357 & 2.7220 & & 2005 & 22.1073 & 28.9807 & 26.0388 & 1.9475 \\
\hline & 2010 & 4.8857 & 14.0768 & 8.8109 & 2.4160 & & 2010 & 25.2166 & 30.3014 & 28.1310 & 1.5564 \\
\hline \multirow[t]{8}{*}{ March } & 1975 & 6.6072 & 15.0204 & 10.1282 & 2.2135 & September & 1975 & 16.5458 & 26.1924 & 20.8361 & 2.7081 \\
\hline & 1980 & 3.7778 & 13.6001 & 7.4684 & 2.5244 & & 1980 & 14.3647 & 24.4542 & 19.1395 & 2.5351 \\
\hline & 1985 & 1.5080 & 13.6020 & 7.9737 & 2.9179 & & 1985 & 15.2840 & 25.1916 & 20.5854 & 2.4419 \\
\hline & 1990 & 5.8160 & 14.7953 & 9.8410 & 2.4662 & & 1990 & 15.4788 & 25.2687 & 19.8176 & 2.4584 \\
\hline & 1995 & 4.9791 & 14.0615 & 8.4624 & 2.4898 & & 1995 & 17.0612 & 25.4172 & 21.0791 & 2.1735 \\
\hline & 2000 & 3.4803 & 12.7911 & 7.1545 & 2.6171 & & 2000 & 16.3220 & 25.8142 & 21.1166 & 2.4484 \\
\hline & 2005 & 5.0723 & 13.9870 & 8.8848 & 2.4185 & & 2005 & 16.4752 & 25.7482 & 20.7710 & 2.5786 \\
\hline & 2010 & 5.8425 & 16.4715 & 10.4517 & 2.3320 & & 2010 & 18.3427 & 25.9256 & 21.7630 & 2.1259 \\
\hline \multirow[t]{8}{*}{ April } & 1975 & 11.4027 & 17.6734 & 13.9034 & 1.7218 & October & 1975 & 11.0174 & 21.0976 & 14.8899 & 2.6129 \\
\hline & 1980 & 8.4474 & 15.4275 & 11.4915 & 2.0839 & & 1980 & 12.3536 & 21.1985 & 16.2249 & 2.3637 \\
\hline & 1985 & 10.4556 & 17.2842 & 13.9654 & 2.0125 & & 1985 & 8.7044 & 19.1918 & 13.0156 & 2.5467 \\
\hline & 1990 & 9.9327 & 17.4434 & 13.1470 & 2.2247 & & 1990 & 11.7813 & 21.7325 & 16.0564 & 2.4525 \\
\hline & 1995 & 8.2844 & 15.9269 & 11.5358 & 2.2356 & & 1995 & 9.2743 & 19.8509 & 13.8540 & 2.5798 \\
\hline & 2000 & 11.7082 & 17.5848 & 14.3388 & 1.8857 & & 2000 & 10.5590 & 20.9450 & 14.9599 & 2.6980 \\
\hline & 2005 & 9.9162 & 17.1927 & 13.1155 & 2.1300 & & 2005 & 9.7082 & 20.0835 & 13.8896 & 2.6384 \\
\hline & 2010 & 9.9424 & 18.6159 & 13.8762 & 2.4406 & & 2010 & 10.2576 & 21.7663 & 14.9154 & 2.9379 \\
\hline \multirow[t]{8}{*}{ May } & 1975 & 13.7103 & 20.7852 & 17.0399 & 2.3309 & November & 1975 & 5.2976 & 16.6203 & 9.3378 & 2.6591 \\
\hline & 1980 & 14.1786 & 20.2122 & 16.8566 & 1.7605 & & 1980 & 7.4070 & 18.1094 & 11.4330 & 2.6440 \\
\hline & 1985 & 15.8079 & 22.5850 & 19.2854 & 2.1122 & & 1985 & 8.4017 & 18.0259 & 12.0326 & 2.3988 \\
\hline & 1990 & 13.3384 & 21.6440 & 17.3523 & 2.5074 & & 1990 & 7.6179 & 18.0775 & 12.0642 & 2.6051 \\
\hline & 1995 & 15.4864 & 21.1762 & 18.3836 & 1.6126 & & 1995 & 1.5022 & 13.8388 & 6.7709 & 3.0380 \\
\hline & 2000 & 13.9277 & 22.0098 & 18.0709 & 2.5511 & & 2000 & 7.1828 & 18.5339 & 11.8420 & 2.5075 \\
\hline & 2005 & 14.9138 & 21.6488 & 18.4084 & 2.1777 & & 2005 & 5.1913 & 15.7904 & 9.1067 & 2.5619 \\
\hline & 2010 & 14.5255 & 22.5254 & 19.3349 & 2.2301 & & 2010 & 9.6838 & 19.5951 & 13.9344 & 2.3800 \\
\hline \multirow[t]{8}{*}{ June } & 1975 & 17.7122 & 24.6564 & 21.2720 & 2.2485 & December & 1975 & -3.2787 & 13.1689 & 3.8009 & 3.8370 \\
\hline & 1980 & 18.1593 & 25.9312 & 21.7894 & 2.0464 & & 1980 & 2.1513 & 13.9154 & 6.7185 & 2.9002 \\
\hline & 1985 & 18.0790 & 26.3428 & 22.1907 & 2.3044 & & 1985 & 2.0959 & 14.5184 & 6.6195 & 3.0085 \\
\hline & 1990 & 17.3079 & 25.9777 & 21.8186 & 2.6735 & & 1990 & 2.4093 & 14.2393 & 7.3080 & 3.0477 \\
\hline & 1995 & 19.8111 & 27.7873 & 24.1458 & 2.4046 & & 1995 & 1.0647 & 14.7638 & 7.1749 & 3.1052 \\
\hline & 2000 & 18.0133 & 27.5768 & 23.1268 & 2.8309 & & 2000 & 1.3021 & 14.4294 & 6.5028 & 3.1720 \\
\hline & 2005 & 17.8073 & 25.9265 & 22.0558 & 2.4529 & & 2005 & 3.0349 & 13.6756 & 7.2157 & 2.8025 \\
\hline & 2010 & 18.0412 & 26.3838 & 22.1602 & 2.4779 & & 2010 & 5.2619 & 15.6738 & 9.0097 & 2.6403 \\
\hline
\end{tabular}


TABLE III

Correlation matrix for precipitation layers.

\begin{tabular}{|c|c|c|c|c|c|c|c|c|}
\hline \multicolumn{9}{|c|}{ CORRELATION MATRIX (Precipitation-January) } \\
\hline Layer(Year) & 1975 & 1980 & 1985 & 1990 & 1995 & 2000 & 2005 & 2010 \\
\hline 1975 & 1.00000 & 0.78846 & 0.78386 & 0.12940 & 0.87703 & 0.75682 & 0.77189 & 0.92382 \\
\hline 1980 & 0.78846 & 1.00000 & 0.86062 & 0.17125 & 0.81056 & 0.69908 & 0.65659 & 0.77735 \\
\hline 1985 & 0.78386 & 0.86062 & 1.00000 & 0.16711 & 0.69942 & 0.63916 & 0.60556 & 0.81675 \\
\hline 1990 & 0.12940 & 0.17125 & 0.16711 & 1.00000 & -0.04847 & 0.39700 & 0.01441 & 0.06176 \\
\hline 1995 & 0.87703 & 0.81056 & 0.69942 & -0.04847 & 1.00000 & 0.55791 & 0.78129 & 0.86215 \\
\hline 2000 & 0.75682 & 0.69908 & 0.63916 & 0.39700 & 0.55791 & 1.00000 & 0.61256 & 0.74714 \\
\hline 2005 & 0.77189 & 0.65659 & 0.60556 & 0.01441 & 0.78129 & 0.61256 & 1.00000 & 0.81718 \\
\hline 2010 & 0.92382 & 0.77735 & 0.81675 & 0.06176 & 0.86215 & 0.74714 & 0.81718 & 1.00000 \\
\hline \multicolumn{9}{|c|}{ CORRELATION MATRIX (Precipitation-February) } \\
\hline Layer(Year) & 1975 & 1980 & 1985 & 1990 & 1995 & 2000 & 2005 & 2010 \\
\hline 1975 & 1.00000 & 0.77978 & 0.71037 & 0.75465 & 0.78511 & 0.32408 & 0.62331 & 0.59694 \\
\hline 1980 & 0.77978 & 1.00000 & 0.70680 & 0.65260 & 0.75107 & 0.18609 & 0.51814 & 0.44678 \\
\hline 1985 & 0.71037 & 0.70680 & 1.00000 & 0.67623 & 0.59647 & 0.25324 & 0.33989 & 0.25617 \\
\hline 1990 & 0.75465 & 0.65260 & 0.67623 & 1.00000 & 0.79150 & 0.64538 & 0.84264 & 0.77847 \\
\hline 1995 & 0.78511 & 0.75107 & 0.59647 & 0.79150 & 1.00000 & 0.50060 & 0.71130 & 0.68328 \\
\hline 2000 & 0.32408 & 0.18609 & 0.25324 & 0.64538 & 0.50060 & 1.00000 & 0.53958 & 0.66191 \\
\hline 2005 & 0.62331 & 0.51814 & 0.33989 & 0.84264 & 0.71130 & 0.53958 & 1.00000 & 0.92908 \\
\hline 2010 & 0.59694 & 0.44678 & 0.25617 & 0.77847 & 0.68328 & 0.66191 & 0.92908 & 1.00000 \\
\hline \multicolumn{9}{|c|}{ CORRELATION MATRIX (Precipitation-March) } \\
\hline Layer(Year) & 1975 & 1980 & 1985 & 1990 & 1995 & 2000 & 2005 & 2010 \\
\hline 1975 & 1.00000 & 0.43577 & 0.43600 & 0.54000 & 0.42494 & 0.60580 & 0.65125 & 0.24777 \\
\hline 1980 & 0.43577 & 1.00000 & 0.48775 & 0.59721 & 0.63492 & 0.69036 & 0.10031 & -0.57255 \\
\hline 1985 & 0.43600 & 0.48775 & 1.00000 & 0.24778 & 0.74793 & 0.23000 & -0.00553 & -0.38343 \\
\hline 1990 & 0.54000 & 0.59721 & 0.24778 & 1.00000 & 0.34694 & 0.57528 & 0.44113 & 0.00851 \\
\hline 1995 & 0.42494 & 0.63492 & 0.74793 & 0.34694 & 1.00000 & 0.51069 & -0.00559 & -0.43359 \\
\hline 2000 & 0.60580 & 0.69036 & 0.23000 & 0.57528 & 0.51069 & 1.00000 & 0.39025 & -0.20299 \\
\hline 2005 & 0.65125 & 0.10031 & -0.00553 & 0.44113 & -0.00559 & 0.39025 & 1.00000 & 0.61318 \\
\hline 2010 & 0.24777 & -0.57255 & -0.38343 & 0.00851 & -0.43359 & -0.20299 & 0.61318 & 1.00000 \\
\hline \multicolumn{9}{|c|}{$\begin{array}{l}\text { CORRELATION MATRIX (Precipitation-April) } \\
\text {. }\end{array}$} \\
\hline Layer(Year) & 1975 & 1980 & 1985 & 1990 & 1995 & 2000 & 2005 & 2010 \\
\hline 1975 & 1.00000 & 0.04649 & -0.29210 & 0.22152 & 0.08140 & -0.09371 & -0.43776 & -0.29386 \\
\hline 1980 & 0.04649 & 1.00000 & 0.20565 & 0.08084 & 0.25487 & -0.06780 & 0.46926 & 0.44613 \\
\hline 1985 & -0.29210 & 0.20565 & 1.00000 & 0.11292 & 0.31988 & 0.64021 & 0.74893 & 0.63354 \\
\hline 1990 & 0.22152 & 0.08084 & 0.11292 & 1.00000 & 0.53602 & 0.27626 & -0.03302 & -0.19927 \\
\hline 1995 & 0.08140 & 0.25487 & 0.31988 & 0.53602 & 1.00000 & 0.62172 & 0.26567 & -0.06769 \\
\hline 2000 & -0.09371 & -0.06780 & 0.64021 & 0.27626 & 0.62172 & 1.00000 & 0.33247 & 0.06834 \\
\hline 2005 & -0.43776 & 0.46926 & 0.74893 & -0.03302 & 0.26567 & 0.33247 & 1.00000 & 0.76531 \\
\hline 2010 & -0.29386 & 0.44613 & 0.63354 & -0.19927 & -0.06769 & 0.06834 & 0.76531 & 1.00000 \\
\hline \multicolumn{9}{|c|}{ CORRELATION MATRIX (Precipitation-May) } \\
\hline Layer(Year) & 1975 & 1980 & 1985 & 1990 & 1995 & 2000 & 2005 & 2010 \\
\hline 1975 & 1.00000 & 0.00014 & 0.51378 & 0.40567 & 0.04118 & 0.58152 & -0.03261 & -0.35965 \\
\hline 1980 & 0.00014 & 1.00000 & -0.14016 & 0.09716 & 0.11290 & 0.16423 & -0.14988 & -0.11414 \\
\hline 1985 & 0.51378 & -0.14016 & 1.00000 & -0.22438 & -0.12253 & -0.13165 & -0.22834 & 0.13157 \\
\hline 1990 & 0.40567 & 0.09716 & -0.22438 & 1.00000 & 0.28300 & 0.86128 & 0.64427 & -0.51083 \\
\hline 1995 & 0.04118 & 0.11290 & -0.12253 & 0.28300 & 1.00000 & 0.19170 & 0.21629 & 0.00804 \\
\hline 2000 & 0.58152 & 0.16423 & -0.13165 & 0.86128 & 0.19170 & 1.00000 & 0.29651 & -0.62850 \\
\hline 2005 & -0.03261 & -0.14988 & -0.22834 & 0.64427 & 0.21629 & 0.29651 & 1.00000 & 0.05753 \\
\hline 2010 & -0.35965 & -0.11414 & 0.13157 & -0.51083 & 0.00804 & -0.62850 & 0.05753 & 1.00000 \\
\hline
\end{tabular}


TABLE III (continuation)

\begin{tabular}{|c|c|c|c|c|c|c|c|c|}
\hline \multicolumn{9}{|c|}{ CORRELATION MATRIX (Precipitation-June) } \\
\hline Layer(Year) & 1975 & 1980 & 1985 & 1990 & 1995 & 2000 & 2005 & 2010 \\
\hline 1975 & 1.00000 & 0.20265 & 0.20593 & 0.28976 & 0.32791 & 0.31104 & 0.10884 & -0.22344 \\
\hline 1980 & 0.20265 & 1.00000 & -0.25895 & 0.25642 & -0.01494 & -0.26385 & 0.30535 & 0.13046 \\
\hline 1985 & 0.20593 & -0.25895 & 1.00000 & 0.61296 & 0.63628 & 0.78552 & 0.42126 & 0.32951 \\
\hline 1990 & 0.28976 & 0.25642 & 0.61296 & 1.00000 & 0.79177 & 0.60613 & 0.66090 & 0.48602 \\
\hline 1995 & 0.32791 & -0.01494 & 0.63628 & 0.79177 & 1.00000 & 0.76352 & 0.57111 & 0.30044 \\
\hline 2000 & 0.31104 & -0.26385 & 0.78552 & 0.60613 & 0.76352 & 1.00000 & 0.27430 & 0.25948 \\
\hline 2005 & 0.10884 & 0.30535 & 0.42126 & 0.66090 & 0.57111 & 0.27430 & 1.00000 & 0.48441 \\
\hline 2010 & -0.22344 & 0.13046 & 0.32951 & 0.48602 & 0.30044 & 0.25948 & 0.48441 & 1.00000 \\
\hline
\end{tabular}

\begin{tabular}{|c|c|c|c|c|c|c|c|c|}
\hline \multicolumn{9}{|c|}{ CORRELATION MATRIX (Precipitation-July) } \\
\hline Layer(Year) & 1975 & 1980 & 1985 & 1990 & 1995 & 2000 & 2005 & 2010 \\
\hline 1975 & 1.00000 & 0.22850 & -0.22994 & 0.71580 & 0.46294 & 0.37854 & 0.62662 & 0.74375 \\
\hline 1980 & 0.22850 & 1.00000 & 0.20738 & 0.21785 & 0.23528 & 0.29285 & 0.10786 & 0.54332 \\
\hline 1985 & -0.22994 & 0.20738 & 1.00000 & 0.11996 & 0.04063 & 0.20946 & 0.10074 & 0.05492 \\
\hline 1990 & 0.71580 & 0.21785 & 0.11996 & 1.00000 & 0.29884 & 0.37246 & 0.57299 & 0.65655 \\
\hline 1995 & 0.46294 & 0.23528 & 0.04063 & 0.29884 & 1.00000 & 0.04565 & 0.39870 & 0.56466 \\
\hline 2000 & 0.37854 & 0.29285 & 0.20946 & 0.37246 & 0.04565 & 1.00000 & 0.55639 & 0.27101 \\
\hline 2005 & 0.62662 & 0.10786 & 0.10074 & 0.57299 & 0.39870 & 0.55639 & 1.00000 & 0.40386 \\
\hline 2010 & 0.74375 & 0.54332 & 0.05492 & 0.65655 & 0.56466 & 0.27101 & 0.40386 & 1.00000 \\
\hline
\end{tabular}

\begin{tabular}{|c|c|c|c|c|c|c|c|c|}
\hline \multicolumn{9}{|c|}{ CORRELATION MATRIX (Precipitation-August) } \\
\hline Layer(Year) & 1975 & 1980 & 1985 & 1990 & 1995 & 2000 & 2005 & 2010 \\
\hline 1975 & 1.00000 & 0.62665 & 0.45484 & 0.33267 & 0.52327 & 0.80480 & 0.47424 & 0.60626 \\
\hline 1980 & 0.62665 & 1.00000 & 0.63235 & 0.23099 & 0.34192 & 0.67653 & 0.49201 & 0.68952 \\
\hline 1985 & 0.45484 & 0.63235 & 1.00000 & 0.27238 & 0.49517 & 0.47897 & 0.36196 & 0.71590 \\
\hline 1990 & 0.33267 & 0.23099 & 0.27238 & 1.00000 & 0.11132 & 0.34003 & 0.01302 & 0.18870 \\
\hline 1995 & 0.52327 & 0.34192 & 0.49517 & 0.11132 & 1.00000 & 0.35925 & 0.67713 & 0.59460 \\
\hline 2000 & 0.80480 & 0.67653 & 0.47897 & 0.34003 & 0.35925 & 1.00000 & 0.51379 & 0.66336 \\
\hline 2005 & 0.47424 & 0.49201 & 0.36196 & 0.01302 & 0.67713 & 0.51379 & 1.00000 & 0.55531 \\
\hline 2010 & 0.60626 & 0.68952 & 0.71590 & 0.18870 & 0.59460 & 0.66336 & 0.55531 & 1.00000 \\
\hline \multicolumn{9}{|c|}{ CORRELATION MATRIX (Precipitation-September) } \\
\hline Layer(Year) & 1975 & 1980 & 1985 & 1990 & 1995 & 2000 & 2005 & 2010 \\
\hline 1975 & 1.00000 & 0.37163 & 0.19678 & 0.59756 & 0.53491 & 0.04033 & 0.02862 & 0.39440 \\
\hline 1980 & 0.37163 & 1.00000 & 0.57914 & 0.66755 & -0.08048 & 0.69922 & 0.38872 & 0.20890 \\
\hline 1985 & 0.19678 & 0.57914 & 1.00000 & 0.36662 & -0.10389 & 0.58103 & 0.36137 & 0.23253 \\
\hline 1990 & 0.59756 & 0.66755 & 0.36662 & 1.00000 & 0.37907 & 0.41984 & 0.03516 & 0.24139 \\
\hline 1995 & 0.53491 & -0.08048 & -0.10389 & 0.37907 & 1.00000 & -0.20455 & -0.19451 & 0.65751 \\
\hline 2000 & 0.04033 & 0.69922 & 0.58103 & 0.41984 & -0.20455 & 1.00000 & 0.52987 & 0.17506 \\
\hline 2005 & 0.02862 & 0.38872 & 0.36137 & 0.03516 & -0.19451 & 0.52987 & 1.00000 & 0.31895 \\
\hline 2010 & 0.39440 & 0.20890 & 0.23253 & 0.24139 & 0.65751 & 0.17506 & 0.31895 & 1.00000 \\
\hline \multicolumn{9}{|c|}{ CORRELATION MATRIX (Precipitation-October) } \\
\hline Layer(Year) & 1975 & 1980 & 1985 & 1990 & 1995 & 2000 & 2005 & 2010 \\
\hline 1975 & 1.00000 & 0.06983 & 0.66618 & -0.34307 & -0.21368 & 0.26494 & 0.62937 & 0.25635 \\
\hline 1980 & 0.06983 & 1.00000 & 0.49524 & 0.12709 & 0.66087 & -0.57686 & 0.44764 & -0.60944 \\
\hline 1985 & 0.66618 & 0.49524 & 1.00000 & -0.08372 & 0.13923 & 0.01497 & 0.61832 & -0.06324 \\
\hline 1990 & -0.34307 & 0.12709 & -0.08372 & 1.00000 & 0.50000 & -0.16595 & -0.20978 & -0.04511 \\
\hline 1995 & -0.21368 & 0.66087 & 0.13923 & 0.50000 & 1.00000 & -0.68396 & 0.06114 & -0.63589 \\
\hline 2000 & 0.26494 & -0.57686 & 0.01497 & -0.16595 & -0.68396 & 1.00000 & -0.10283 & 0.74704 \\
\hline 2005 & 0.62937 & 0.44764 & 0.61832 & -0.20978 & 0.06114 & -0.10283 & 1.00000 & -0.27208 \\
\hline 2010 & 0.25635 & -0.60944 & -0.06324 & -0.04511 & -0.63589 & 0.74704 & -0.27208 & 1.00000 \\
\hline
\end{tabular}


TABLE III (continuation)

\begin{tabular}{|c|c|c|c|c|c|c|c|c|}
\hline \multicolumn{9}{|c|}{ CORRELATION MATRIX (Precipitation-November) } \\
\hline Layer(Year) & 1975 & 1980 & 1985 & 1990 & 1995 & 2000 & 2005 & 2010 \\
\hline 1975 & 1.00000 & 0.79762 & 0.65602 & 0.45331 & 0.41620 & 0.62822 & 0.79794 & 0.72336 \\
\hline 1980 & 0.79762 & 1.00000 & 0.77541 & 0.57684 & 0.59180 & 0.52085 & 0.72144 & 0.68886 \\
\hline 1985 & 0.65602 & 0.77541 & 1.00000 & 0.63039 & 0.56208 & 0.64761 & 0.72962 & 0.73200 \\
\hline 1990 & 0.45331 & 0.57684 & 0.63039 & 1.00000 & 0.34262 & 0.47725 & 0.34958 & 0.29150 \\
\hline 1995 & 0.41620 & 0.59180 & 0.56208 & 0.34262 & 1.00000 & 0.45066 & 0.43236 & 0.34430 \\
\hline 2000 & 0.62822 & 0.52085 & 0.64761 & 0.47725 & 0.45066 & 1.00000 & 0.64668 & 0.55346 \\
\hline 2005 & 0.79794 & 0.72144 & 0.72962 & 0.34958 & 0.43236 & 0.64668 & 1.00000 & 0.85486 \\
\hline 2010 & 0.72336 & 0.68886 & 0.73200 & 0.29150 & 0.34430 & 0.55346 & 0.85486 & 1.00000 \\
\hline \multicolumn{9}{|c|}{ CORRELATION MATRIX (Precipitation-December) } \\
\hline Layer(Year) & 1975 & 1980 & 1985 & 1990 & 1995 & 2000 & 2005 & 2010 \\
\hline 1975 & 1.00000 & 0.79379 & 0.25259 & 0.81574 & 0.78905 & 0.50197 & 0.86966 & 0.64845 \\
\hline 1980 & 0.79379 & 1.00000 & 0.31704 & 0.90808 & 0.90569 & 0.39818 & 0.78649 & 0.80757 \\
\hline 1985 & 0.25259 & 0.31704 & 1.00000 & 0.22520 & 0.48945 & 0.76838 & 0.31490 & 0.26742 \\
\hline 1990 & 0.81574 & 0.90808 & 0.22520 & 1.00000 & 0.85097 & 0.42111 & 0.74077 & 0.78907 \\
\hline 1995 & 0.78905 & 0.90569 & 0.48945 & 0.85097 & 1.00000 & 0.56697 & 0.77087 & 0.77156 \\
\hline 2000 & 0.50197 & 0.39818 & 0.76838 & 0.42111 & 0.56697 & 1.00000 & 0.52801 & 0.38538 \\
\hline 2005 & 0.86966 & 0.78649 & 0.31490 & 0.74077 & 0.77087 & 0.52801 & 1.00000 & 0.65563 \\
\hline 2010 & 0.64845 & 0.80757 & 0.26742 & 0.78907 & 0.77156 & 0.38538 & 0.65563 & 1.00000 \\
\hline
\end{tabular}

TABLE IV

Correlation matrix for temperature layers.

\begin{tabular}{|c|c|c|c|c|c|c|c|c|}
\hline \multicolumn{9}{|c|}{ CORRELATION MATRIX (Temperature-January) } \\
\hline Layer(Year) & 1975 & 1980 & 1985 & 1990 & 1995 & 2000 & 2005 & 2010 \\
\hline 1975 & 1.00000 & 0.99001 & 0.98543 & 0.99064 & 0.98531 & 0.99384 & 0.99098 & 0.97266 \\
\hline 1980 & 0.99001 & 1.00000 & 0.99524 & 0.98171 & 0.99200 & 0.99217 & 0.99463 & 0.97417 \\
\hline 1985 & 0.98543 & 0.99524 & 1.00000 & 0.97166 & 0.99482 & 0.98869 & 0.99503 & 0.97539 \\
\hline 1990 & 0.99064 & 0.98171 & 0.97166 & 1.00000 & 0.97136 & 0.98582 & 0.97629 & 0.95152 \\
\hline 1995 & 0.98531 & 0.99200 & 0.99482 & 0.97136 & 1.00000 & 0.98977 & 0.99353 & 0.97975 \\
\hline 2000 & 0.99384 & 0.99217 & 0.98869 & 0.98582 & 0.98977 & 1.00000 & 0.99041 & 0.97014 \\
\hline 2005 & 0.99098 & 0.99463 & 0.99503 & 0.97629 & 0.99353 & 0.99041 & 1.00000 & 0.98280 \\
\hline 2010 & 0.97266 & 0.97417 & 0.97539 & 0.95152 & 0.97975 & 0.97014 & 0.98280 & 1.00000 \\
\hline \multicolumn{9}{|c|}{ CORRELATION MATRIX (Temperature-February) } \\
\hline Layer(Year) & 1975 & 1980 & 1985 & 1990 & 1995 & 2000 & 2005 & 2010 \\
\hline 1975 & 1.00000 & 0.99420 & 0.99281 & 0.98748 & 0.98275 & 0.96240 & 0.98388 & 0.97305 \\
\hline 1980 & 0.99420 & 1.00000 & 0.99591 & 0.99286 & 0.98342 & 0.97515 & 0.98280 & 0.96708 \\
\hline 1985 & 0.99281 & 0.99591 & 1.00000 & 0.99162 & 0.98447 & 0.97700 & 0.98697 & 0.97381 \\
\hline 1990 & 0.98748 & 0.99286 & 0.99162 & 1.00000 & 0.98965 & 0.98378 & 0.98806 & 0.97410 \\
\hline 1995 & 0.98275 & 0.98342 & 0.98447 & 0.98965 & 1.00000 & 0.98594 & 0.98194 & 0.98254 \\
\hline 2000 & 0.96240 & 0.97515 & 0.97700 & 0.98378 & 0.98594 & 1.00000 & 0.96256 & 0.95804 \\
\hline 2005 & 0.98388 & 0.98280 & 0.98697 & 0.98806 & 0.98194 & 0.96256 & 1.00000 & 0.98685 \\
\hline 2010 & 0.97305 & 0.96708 & 0.97381 & 0.97410 & 0.98254 & 0.95804 & 0.98685 & 1.00000 \\
\hline \multicolumn{9}{|c|}{ CORRELATION MATRIX (Temperature-March) } \\
\hline Layer(Year) & 1975 & 1980 & 1985 & 1990 & 1995 & 2000 & 2005 & 2010 \\
\hline 1975 & 1.00000 & 0.96776 & 0.93293 & 0.96541 & 0.98026 & 0.97802 & 0.96322 & 0.91016 \\
\hline 1980 & 0.96776 & 1.00000 & 0.97682 & 0.97817 & 0.98798 & 0.98687 & 0.99252 & 0.96439 \\
\hline 1985 & 0.93293 & 0.97682 & 1.00000 & 0.97683 & 0.95402 & 0.95607 & 0.96738 & 0.93709 \\
\hline 1990 & 0.96541 & 0.97817 & 0.97683 & 1.00000 & 0.97132 & 0.97380 & 0.97428 & 0.93204 \\
\hline 1995 & 0.98026 & 0.98798 & 0.95402 & 0.97132 & 1.00000 & 0.99459 & 0.98740 & 0.93968 \\
\hline 2000 & 0.97802 & 0.98687 & 0.95607 & 0.97380 & 0.99459 & 1.00000 & 0.98191 & 0.93228 \\
\hline 2005 & 0.96322 & 0.99252 & 0.96738 & 0.97428 & 0.98740 & 0.98191 & 1.00000 & 0.96909 \\
\hline 2010 & 0.91016 & 0.96439 & 0.93709 & 0.93204 & 0.93968 & 0.93228 & 0.96909 & 1.00000 \\
\hline
\end{tabular}


TABLE IV (continuation)

\begin{tabular}{|c|c|c|c|c|c|c|c|c|}
\hline \multicolumn{9}{|c|}{ CORRELATION MATRIX (Temperature-April) } \\
\hline Layer(Year) & 1975 & 1980 & 1985 & 1990 & 1995 & 2000 & 2005 & 2010 \\
\hline 1975 & 1.00000 & 0.97750 & 0.97998 & 0.97378 & 0.97661 & 0.96197 & 0.96881 & 0.94823 \\
\hline 1980 & 0.97750 & 1.00000 & 0.98631 & 0.98860 & 0.99361 & 0.96976 & 0.98274 & 0.97421 \\
\hline 1985 & 0.97998 & 0.98631 & 1.00000 & 0.98679 & 0.98503 & 0.97772 & 0.98419 & 0.96170 \\
\hline 1990 & 0.97378 & 0.98860 & 0.98679 & 1.00000 & 0.99253 & 0.98757 & 0.99332 & 0.98398 \\
\hline 1995 & 0.97661 & 0.99361 & 0.98503 & 0.99253 & 1.00000 & 0.97755 & 0.98891 & 0.97271 \\
\hline 2000 & 0.96197 & 0.96976 & 0.97772 & 0.98757 & 0.97755 & 1.00000 & 0.98612 & 0.96112 \\
\hline 2005 & 0.96881 & 0.98274 & 0.98419 & 0.99332 & 0.98891 & 0.98612 & 1.00000 & 0.97864 \\
\hline 2010 & 0.94823 & 0.97421 & 0.96170 & 0.98398 & 0.97271 & 0.96112 & 0.97864 & 1.00000 \\
\hline
\end{tabular}

CORRELATION MATRIX (Temperature-May)

\begin{tabular}{lllllllll} 
Layer(Year) & $\mathbf{1 9 7 5}$ & $\mathbf{1 9 8 0}$ & $\mathbf{1 9 8 5}$ & $\mathbf{1 9 9 0}$ & $\mathbf{1 9 9 5}$ & $\mathbf{2 0 0 0}$ & $\mathbf{2 0 0 5}$ & $\mathbf{2 0 1 0}$ \\
$\mathbf{1 9 7 5}$ & 1.00000 & 0.98754 & 0.99057 & 0.97503 & 0.96946 & 0.98393 & 0.98151 & 0.97436 \\
$\mathbf{1 9 8 0}$ & 0.98754 & 1.00000 & 0.99004 & 0.97957 & 0.98300 & 0.98179 & 0.98569 & 0.97928 \\
$\mathbf{1 9 8 5}$ & 0.99057 & 0.99004 & 1.00000 & 0.97169 & 0.98487 & 0.97795 & 0.97774 & 0.97943 \\
$\mathbf{1 9 9 0}$ & 0.97503 & 0.97957 & 0.97169 & 1.00000 & 0.97585 & 0.99414 & 0.98976 & 0.96744 \\
$\mathbf{1 9 9 5}$ & 0.96946 & 0.98300 & 0.98487 & 0.97585 & 1.00000 & 0.97536 & 0.97691 & 0.97110 \\
$\mathbf{2 0 0 0}$ & 0.98393 & 0.98179 & 0.97795 & 0.99414 & 0.97536 & 1.00000 & 0.98905 & 0.97046 \\
$\mathbf{2 0 0 5}$ & 0.98151 & 0.98569 & 0.97774 & 0.98976 & 0.97691 & 0.98905 & 1.00000 & 0.96891 \\
$\mathbf{2 0 1 0}$ & 0.97436 & 0.97928 & 0.97943 & 0.96744 & 0.97110 & 0.97046 & 0.96891 & 1.00000 \\
\hline \hline
\end{tabular}

\begin{tabular}{|c|c|c|c|c|c|c|c|c|}
\hline \multicolumn{9}{|c|}{ CORRELATION MATRIX (Temperature-June) } \\
\hline Layer(Year) & 1975 & 1980 & 1985 & 1990 & 1995 & 2000 & 2005 & 2010 \\
\hline 1975 & 1.00000 & 0.95346 & 0.98094 & 0.97460 & 0.97601 & 0.97138 & 0.96753 & 0.97943 \\
\hline 1980 & 0.95346 & 1.00000 & 0.98204 & 0.98186 & 0.95395 & 0.97538 & 0.98243 & 0.94961 \\
\hline 1985 & 0.98094 & 0.98204 & 1.00000 & 0.99084 & 0.97684 & 0.99073 & 0.99018 & 0.97364 \\
\hline 1990 & 0.97460 & 0.98186 & 0.99084 & 1.00000 & 0.98152 & 0.98635 & 0.98838 & 0.97215 \\
\hline 1995 & 0.97601 & 0.95395 & 0.97684 & 0.98152 & 1.00000 & 0.96700 & 0.96597 & 0.95207 \\
\hline 2000 & 0.97138 & 0.97538 & 0.99073 & 0.98635 & 0.96700 & 1.00000 & 0.98993 & 0.96894 \\
\hline 2005 & 0.96753 & 0.98243 & 0.99018 & 0.98838 & 0.96597 & 0.98993 & 1.00000 & 0.96989 \\
\hline 2010 & 0.97943 & 0.94961 & 0.97364 & 0.97215 & 0.95207 & 0.96894 & 0.96989 & 1.00000 \\
\hline \multicolumn{9}{|c|}{ CORRELATION MATRIX (Temperature-July) } \\
\hline Layer(Year) & 1975 & 1980 & 1985 & 1990 & 1995 & 2000 & 2005 & 2010 \\
\hline 1975 & 1.00000 & 0.97647 & 0.98897 & 0.99041 & 0.97678 & 0.98756 & 0.98871 & 0.97928 \\
\hline 1980 & 0.97647 & 1.00000 & 0.96587 & 0.97782 & 0.93391 & 0.96840 & 0.96744 & 0.95022 \\
\hline 1985 & 0.98897 & 0.96587 & 1.00000 & 0.98248 & 0.98509 & 0.97307 & 0.98407 & 0.97415 \\
\hline 1990 & 0.99041 & 0.97782 & 0.98248 & 1.00000 & 0.95961 & 0.98519 & 0.98069 & 0.97109 \\
\hline 1995 & 0.97678 & 0.93391 & 0.98509 & 0.95961 & 1.00000 & 0.96299 & 0.98156 & 0.96335 \\
\hline 2000 & 0.98756 & 0.96840 & 0.97307 & 0.98519 & 0.96299 & 1.00000 & 0.98577 & 0.96678 \\
\hline 2005 & 0.98871 & 0.96744 & 0.98407 & 0.98069 & 0.98156 & 0.98577 & 1.00000 & 0.96754 \\
\hline 2010 & 0.97928 & 0.95022 & 0.97415 & 0.97109 & 0.96335 & 0.96678 & 0.96754 & 1.00000 \\
\hline \multicolumn{9}{|c|}{ CORRELATION MATRIX (Temperature-August) } \\
\hline Layer(Year) & 1975 & 1980 & 1985 & 1990 & 1995 & 2000 & 2005 & 2010 \\
\hline 1975 & 1.00000 & 0.98710 & 0.98710 & 0.98982 & 0.98119 & 0.98817 & 0.98214 & 0.96580 \\
\hline 1980 & 0.98710 & 1.00000 & 0.98525 & 0.98710 & 0.97927 & 0.98774 & 0.97624 & 0.95483 \\
\hline 1985 & 0.98710 & 0.98525 & 1.00000 & 0.98983 & 0.98460 & 0.99000 & 0.98436 & 0.95388 \\
\hline 1990 & 0.98982 & 0.98710 & 0.98983 & 1.00000 & 0.98274 & 0.99293 & 0.98298 & 0.96703 \\
\hline 1995 & 0.98119 & 0.97927 & 0.98460 & 0.98274 & 1.00000 & 0.98346 & 0.98776 & 0.94175 \\
\hline 2000 & 0.98817 & 0.98774 & 0.99000 & 0.99293 & 0.98346 & 1.00000 & 0.99239 & 0.97248 \\
\hline 2005 & 0.98214 & 0.97624 & 0.98436 & 0.98298 & 0.98776 & 0.99239 & 1.00000 & 0.96056 \\
\hline 2010 & 0.96580 & 0.95483 & 0.95388 & 0.96703 & 0.94175 & 0.97248 & 0.96056 & 1.00000 \\
\hline
\end{tabular}


TABLE IV (continuation)

\begin{tabular}{|c|c|c|c|c|c|c|c|c|}
\hline \multicolumn{9}{|c|}{ CORRELATION MATRIX (Temperature-September) } \\
\hline Layer(Year) & 1975 & 1980 & 1985 & 1990 & 1995 & 2000 & 2005 & 2010 \\
\hline 1975 & 1.00000 & 0.98432 & 0.98128 & 0.98609 & 0.97975 & 0.98318 & 0.98589 & 0.99125 \\
\hline 1980 & 0.98432 & 1.00000 & 0.99155 & 0.98712 & 0.98378 & 0.98371 & 0.97888 & 0.97036 \\
\hline 1985 & 0.98128 & 0.99155 & 1.00000 & 0.98127 & 0.97791 & 0.98163 & 0.97309 & 0.96714 \\
\hline 1990 & 0.98609 & 0.98712 & 0.98127 & 1.00000 & 0.98041 & 0.97355 & 0.97007 & 0.98024 \\
\hline 1995 & 0.97975 & 0.98378 & 0.97791 & 0.98041 & 1.00000 & 0.99027 & 0.98815 & 0.96733 \\
\hline 2000 & 0.98318 & 0.98371 & 0.98163 & 0.97355 & 0.99027 & 1.00000 & 0.99254 & 0.97132 \\
\hline 2005 & 0.98589 & 0.97888 & 0.97309 & 0.97007 & 0.98815 & 0.99254 & 1.00000 & 0.97438 \\
\hline 2010 & 0.99125 & 0.97036 & 0.96714 & 0.98024 & 0.96733 & 0.97132 & 0.97438 & 1.00000 \\
\hline \multicolumn{9}{|c|}{ CORRELATION MATRIX (Temperature-October) } \\
\hline Layer(Year) & 1975 & 1980 & 1985 & 1990 & 1995 & 2000 & 2005 & 2010 \\
\hline 1975 & 1.00000 & 0.98886 & 0.99071 & 0.98472 & 0.99004 & 0.99062 & 0.99542 & 0.98544 \\
\hline 1980 & 0.98886 & 1.00000 & 0.97972 & 0.98269 & 0.98005 & 0.98243 & 0.98307 & 0.97214 \\
\hline 1985 & 0.99071 & 0.97972 & 1.00000 & 0.98881 & 0.99341 & 0.99234 & 0.98544 & 0.97534 \\
\hline 1990 & 0.98472 & 0.98269 & 0.98881 & 1.00000 & 0.98608 & 0.98709 & 0.97632 & 0.97474 \\
\hline 1995 & 0.99004 & 0.98005 & 0.99341 & 0.98608 & 1.00000 & 0.99196 & 0.98567 & 0.97342 \\
\hline 2000 & 0.99062 & 0.98243 & 0.99234 & 0.98709 & 0.99196 & 1.00000 & 0.98698 & 0.98191 \\
\hline 2005 & 0.99542 & 0.98307 & 0.98544 & 0.97632 & 0.98567 & 0.98698 & 1.00000 & 0.98408 \\
\hline 2010 & 0.98544 & 0.97214 & 0.97534 & 0.97474 & 0.97342 & 0.98191 & 0.98408 & 1.00000 \\
\hline \multicolumn{9}{|c|}{ CORRELATION MATRIX (Temperature-November) } \\
\hline Layer(Year) & 1975 & 1980 & 1985 & 1990 & 1995 & 2000 & 2005 & 2010 \\
\hline 1975 & 1.00000 & 0.98838 & 0.99165 & 0.97041 & 0.98973 & 0.96630 & 0.99328 & 0.94459 \\
\hline 1980 & 0.98838 & 1.00000 & 0.98876 & 0.98825 & 0.97959 & 0.97029 & 0.98830 & 0.96251 \\
\hline 1985 & 0.99165 & 0.98876 & 1.00000 & 0.97865 & 0.98934 & 0.97822 & 0.98729 & 0.95602 \\
\hline 1990 & 0.97041 & 0.98825 & 0.97865 & 1.00000 & 0.95958 & 0.97309 & 0.97731 & 0.97502 \\
\hline 1995 & 0.98973 & 0.97959 & 0.98934 & 0.95958 & 1.00000 & 0.96471 & 0.98812 & 0.93409 \\
\hline 2000 & 0.96630 & 0.97029 & 0.97822 & 0.97309 & 0.96471 & 1.00000 & 0.96795 & 0.96403 \\
\hline 2005 & 0.99328 & 0.98830 & 0.98729 & 0.97731 & 0.98812 & 0.96795 & 1.00000 & 0.95475 \\
\hline 2010 & 0.94459 & 0.96251 & 0.95602 & 0.97502 & 0.93409 & 0.96403 & 0.95475 & 1.00000 \\
\hline \multicolumn{9}{|c|}{ CORRELATION MATRIX (Temperature-December) } \\
\hline Layer(Year) & 1975 & 1980 & 1985 & 1990 & 1995 & 2000 & 2005 & 2010 \\
\hline 1975 & 1.00000 & 0.98203 & 0.98128 & 0.98008 & 0.98588 & 0.98097 & 0.96599 & 0.96537 \\
\hline 1980 & 0.98203 & 1.00000 & 0.99205 & 0.99665 & 0.98918 & 0.99443 & 0.99171 & 0.97762 \\
\hline 1985 & 0.98128 & 0.99205 & 1.00000 & 0.98926 & 0.98818 & 0.99459 & 0.98883 & 0.98108 \\
\hline 1990 & 0.98008 & 0.99665 & 0.98926 & 1.00000 & 0.98819 & 0.99248 & 0.99313 & 0.98019 \\
\hline 1995 & 0.98588 & 0.98918 & 0.98818 & 0.98819 & 1.00000 & 0.98769 & 0.98406 & 0.98327 \\
\hline 2000 & 0.98097 & 0.99443 & 0.99459 & 0.99248 & 0.98769 & 1.00000 & 0.98867 & 0.97993 \\
\hline 2005 & 0.96599 & 0.99171 & 0.98883 & 0.99313 & 0.98406 & 0.98867 & 1.00000 & 0.98158 \\
\hline 2010 & 0.96537 & 0.97762 & 0.98108 & 0.98019 & 0.98327 & 0.97993 & 0.98158 & 1.00000 \\
\hline
\end{tabular}

( 72 for precipitation and 72 for temperature). Figures 6 and 7 show the cell statistics of the precipitation and temperature for the month of January.

Here, range layers give the most important information. Range layers indicate the difference between the largest and the smallest value of the inputs. When examining the range layers, it was understood that the month with largest changes is January for precipitation and November for temperature. 

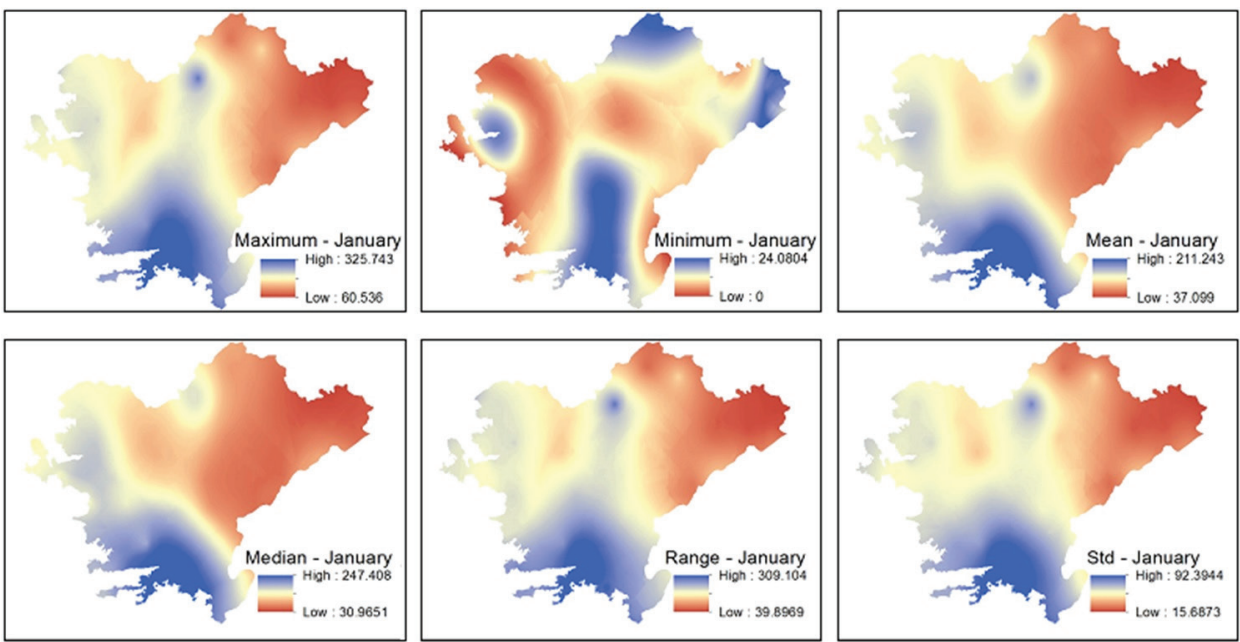

Figure 6 - Cell statistics of the precipitation for the month of January.
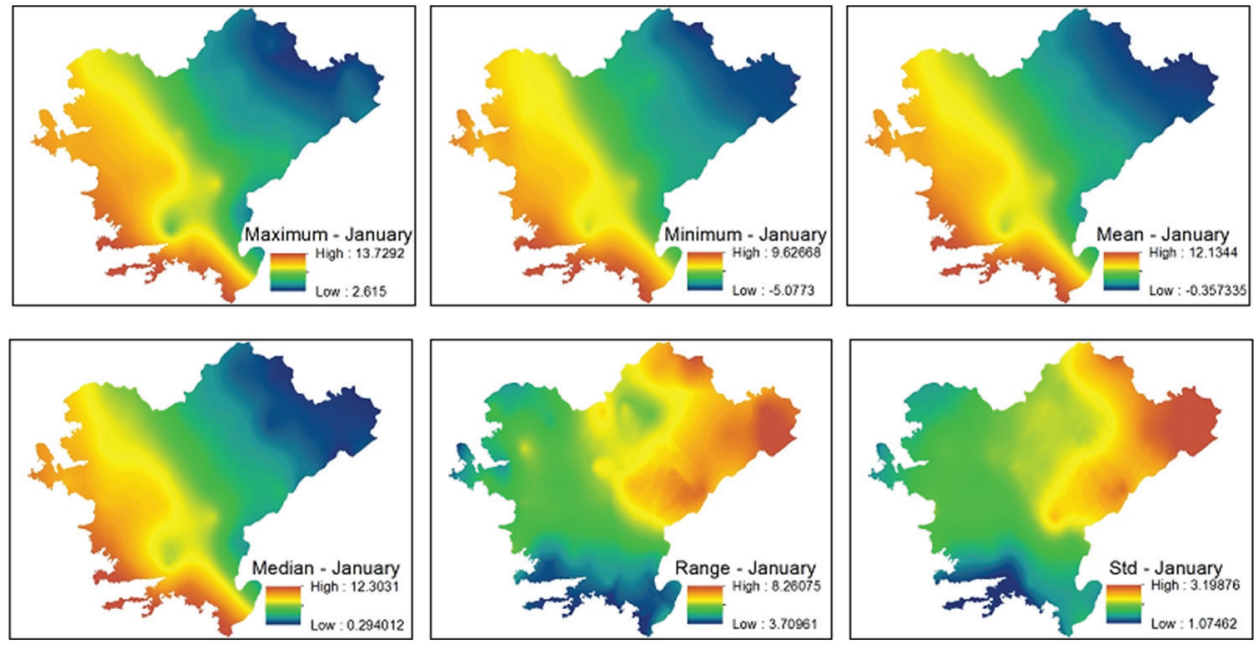

Figure 7 - Cell statistics of the temperature for the month of January.

\section{CONCLUSIONS}

Meteorological data are required in many fields such as environment, agriculture and management of natural disasters where spatial data are used. But meteorological data are generally available from a limited number of stations. For this reason, interpolation techniques are used to obtain complete surface information. In recent years, depending on the technological developments in computer and GIS, geostatistical methods are used in order to determine the spatial distribution of meteorological data and the Ordinary Kriging method is nowadays a preferable option in the literature. Unlike the deterministic methods, geostatistical interpolation techniques also utilize the statistical properties of the measured points. In geostatistical techniques the autocorrelation among the measured points is determined and spatial configuration of the sampling points around the estimation point is taken into consideration.

In this study, spatial distributions of precipitation and temperature of the Aegean Region in Turkey for years 1975, 1980, 1985, 1990, 1995, 2000, 2005 and 2010 in 5-year periods were determined by the Ordinary 
Kriging method. The time series of monthly precipitation and temperature data from 98 meteorological stations were used for the Ordinary Kriging. To evaluate and interpret the results, multivariate statistics (band collection) and cell statistics were applied for the monthly precipitation and temperature layer series. The results revealed that a significant change in precipitation regime in the Aegean Region was occurred. It is necessary to pay attention to this change because of multiple environmental effects of the climate changes. In the following studies, prediction of the future trends and determination of the effects of these changes on nature and human health are required.

\section{REFERENCES}

BORGA M AND VIZZACCARO A. 1996. On the interpolation of hydrologic variables: Formal equivalence of multiquadratic surface fitting and kriging. J Hydrol 195: 160-171.

BURROUGH PA AND MCDONNELL RA. 1998. Principles of Geographical Information Systems: Spatial Information Systems and Geostatistics. NewYork: Oxford University Press.

ESRI. 2014a. ArcGIS Resources - ArcGIS Help 10.2\&10.2.1, http://resources.arcgis.com/en/help/main/10.2/ (accessed 03.03.2014).

ESRI. 2014b. Statistical Analysis, http://www.esri.com/software/arcgis/extensions/spatialanalyst/key-features/statistical (accessed 12.02.2014).

GMS USER MANUAL. 2012. http://gmsdocs.aquaveo.com.s3.amazonaws.com/GMS_8.3_User_Manual.pdf (accessed 03.03.2014).

HOFIERKA J, PARAJKA J, MITASOVA H AND MITAS L. 2002. Multivariate interpolation of precipitation using regularized spline with tension. Trans GIS 6(2): 135-150.

KROPP S. 2015. Climate change and risk of flooding in Germany: Consequences for property values. In: Hepperle E, DixonGough R, Mansberger R, Paulsson J, Reuter F and Yilmaz M (Eds), Challenges for Governance Structures in Urban and Regional Development. ETH Zürich, p. 155-160.

LI J AND HEAP AD. 2008. A Review of Spatial Interpolation Methods for Environmental Scientists. Geoscience Australia Record 2008/23.

MATTHEWS SA. 2002. ArcGIS Geostatistical Analyst, GIS Resource Document 02-19.

NATIONAL ACADEMY OF SCIENCES 2009. Ecological Impacts of Climate Change, USA.

OZCAGLAR A. 2014. Aegean Region, http://www.geography.humanity.ankara.edu.tr/ders_notu/cog_330.pdf (accessed 02.03.2014).

PRASAD R, DIXIT A, MALHOTRA PK AND GUPTA VK. 2007. Geoinformatics in precision farming: An overview. In: Singh AK and Chopra UK (Eds), Geoinformatics Applications in Agriculture. New Delhi: New India Publishing Agency, p. $39-78$.

SENSOY S, DEMIRCAN M, ULUPINAR Y, BALTA I. 2008. Climate of Turkey, http://www.mgm.gov.tr/files/en-us/ climateofturkey.pdf (accessed 02.03.2015).

SHEFFIELD J AND WOOD EF. 2012. Drought in the 21st Century. In: Drought: Past Problems and Future Scenarios. Routledge: Taylor \& Francis, Chapter 8.

VERTICAL MAPPER USER GUIDE. 2008. http://reference.mapinfo.com/software/vertical_mapper/english/3_5/ VerticalMapperUserGuide.pdf (accessed 01.04.2015).

WACKERNAGEL H. 2003. Multivariate Geostatistics: An Introduction with Applications. Berlin : Springer-Verlag.

WALLER LA AND GOTWAY CA. 2004. Applied Spatial Statistics for Public Health Data. New Jersey: J Wiley \& Sons.

WANG M, AIRHIHENBUWA CO AND NNADI-OKOLO E. 1990. Data analysis and selection of statistical methods. In: NnadiOkolo E (Ed), Health Research Design and Methodology. Boca Raton: CRC Press, Chapter 4, p. 149-228.

WATERS NM. 1997. Spatial Interpolation, http://www.geog.ubc.ca/courses/klink/gis.notes/ncgia/u40.html\#SEC40.3.3 (accessed 12.03.2014).

WEBSTER R AND OLIVER MA. 2007. Geostatistics for Environmental Scientist. Chichester: J Wiley \& Sons. 